\title{
Charge Transfer Mechanism and Spatial Density Correlation of Electronic States of Excited Zinc $\left(3 d^{9}\right)$ Films
}

\author{
Li Chen*, Mitsugi Hamasaki, Hirotaka Manaka, Kozo Obara \\ Country Graduate School of Science and Engineering, Kagoshima University, Kagoshima, Japan \\ Email: chenli2009@live.jp
}

Received 14 February 2014; revised 10 March 2014; accepted 18 March 2014

Copyright (C) 2014 by authors and Scientific Research Publishing Inc.

This work is licensed under the Creative Commons Attribution International License (CC BY). http://creativecommons.org/licenses/by/4.0/

(c) (i) Open Access

\begin{abstract}
In material science, half filled 3d orbital of transition metals is essentially an important factor controlling characteristics of alloys and compounds. This paper presents a result of the challenge of excitation of inner-core electron system with long lifetime of zinc films. The advanced zinc films with excited inner-core electron, $3 d^{n}(n=9,8)$. We report experimental results of XPS measurements of 9 points in the sample along vertical direction, respectively. The most pronounced futures are existence of satellites, which are about $4 \mathrm{eV}$ higher than the main lines. According to the charge transfer mechanism proposed by A. Kotani and K. Okada, it was clarified that the origins of these peaks are $\underline{\mathbf{c}} 3 \mathrm{~d}^{9} \underline{L}$ for the main peak and $\underline{\mathbf{c}} 3 \mathrm{~d}^{9}$ for the satellite, respectively. From the energy difference, $\delta E_{B}$, and peak intensity ratio, $I_{+} / I_{-}$, between $\underline{\mathbf{c}} 3 d^{9}$ and $\underline{\mathbf{c}} 3 \mathrm{~d}^{10} \underline{L}$, the energy for charge transfer, $\Delta$, and mixing energy, $T$, were estimated. In the region where the intensity of $\underline{c} 3 d^{10} \underline{L}$ becomes large, $\Delta$ becomes small, $1.2<\Delta<2.7$, and $T$ becomes small, too, $0.1<T<0.9$, respectively. In this calculation, we supposed $U_{\mathrm{dc}}=5.5 \mathrm{eV}$ and $U_{\mathrm{dd}}=5.5 \mathrm{eV}$. In the analysis along vertical direction,

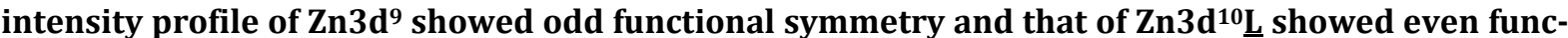
tional symmetry. Only the intensity profile of $\mathrm{C1s}(288 \mathrm{eV})$ showed the same spatial correlation with $\mathrm{Zn3d}^{9}$. In our experiment, the sample also showed high mobility of the constituting elements.

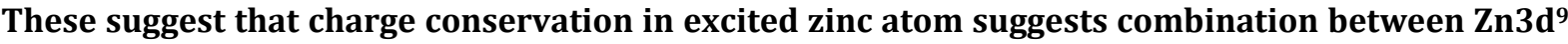
and $\mathrm{C}^{2-}$
\end{abstract}

\section{Keywords}

XPS, Zn3d ${ }^{9}$, Charge Transfer, Spatial Symmetry of Excited States

\footnotetext{
"Corresponding author.
} 


\section{Introduction}

In developing new materials, there are nanotechnology, researching and development of composite materials, the surface material modification and so on. However, these methods are controlling the outer shell electrons, but in material creation, just controlling the outer shell electrons is insufficient. Our research is exciting inner shell electrons of materials (Zinc). This is the way that excitation can affect the nature and performance of materials. There are some important elements in the formation of excimer such as $\mathrm{Cu}$, $\mathrm{Zn}$ and Ga. In particular they have been investigated by many researchers, which just proofed the existence of inner-shell holes and the lifetime of atomic excitation state with very short lifetime [1] [2]. Although, our research is creating excited states with the permanent lifetime and learning the mechanism formation processes. The quantum dynamic approach to the condensation process of excited zinc films by ion-recombination has been concluded by M. Hamasaki and M. Obara [3] [4]. In this paper, the permanent lifetime of excited zinc thin films also has been substantiated by XPS.

\section{Sample Preparation}

The experiment apparatus which was developed just by our research group is an integral evaporation system with transmission electron spectroscopy evaluation (Electron-assisted PVD). Detailed sample preparation procedure has been published in [3] [4]. Main futures are followings: 1) substrate is $6.5^{\varphi}$ mm sapphire enclosed by gold electrode; 2) the surface of sapphire was irradiated with electrons of constant energy; 4) Background pressure is $10^{-5} \mathrm{~Pa}$; 5) substrate temperature is from room temperature $30^{\circ} \mathrm{C}$ to $100^{\circ} \mathrm{C}$; 6) The incident angle of electrons was $45^{\circ}$ from the substrate surface. Then the $0.1 \mathrm{~g}$ zinc atoms were deposited on the insulate area from the effusion cell at $600^{\circ} \mathrm{C}$. The energy dependence of condensed materials was measured. Discrete energy dependence was observed at $10 \mathrm{eV}, 90 \mathrm{eV}, 100 \mathrm{eV}, 140 \mathrm{eV}$, and $230 \mathrm{eV}$. These energies are related to the binding energies of zinc atom, 3d (10 eV), 3p (90 eV), and 3s $(140 \mathrm{eV})$. Observed discreet energy dependence was classified into single excitation and double excitation. Since initial condition of the ion-recombination process is $\mathrm{Zn}^{+}$ and $\mathrm{Zn}^{-}$, transition processes should depend on the selection rule, $\Delta l= \pm 1$ [5]. Observed strong diffuse scattering intensity and enhancement of the intensity of Bragg diffraction are related to the transition at $\mathrm{Zn}^{+}$and $\mathrm{Zn}^{-}$, respectively. The sample shown in Figure 1 was deposited at $100^{\circ} \mathrm{C}$ and $230 \mathrm{eV}$.

We discussed experimental results of XPS measurements of 9 points in the sample with $6.5^{\varphi}$ mm along vertical and horizontal direction, respectively, and it's different between vertical and horizontal direction. In this paper, we report the experimental results at vertical. The direction of the incident electron for the surface bias and zinc ionization is parallel to the horizontal direction. Zinc films could conclude $\mathrm{Zn}$ what is the reaching object element, $\mathrm{Al}$ (substrate of the special excited zinc film is constituted by sapphire, $\mathrm{Al}_{2} \mathrm{O}_{3}$ ), Si (the effusion cell is constituted by $\left.\mathrm{SiO}_{2}\right), \mathrm{C}$ (adsorption from environment) and $\mathrm{O}\left(\mathrm{Al}_{2} \mathrm{O}_{3}, \mathrm{SiO}_{2}\right.$ and adsorption from environment). The standard point of these data is $\mathrm{Au}_{4} \mathrm{f}_{7 / 2}(84.0 \mathrm{eV}), \mathrm{Au}_{5 / 2}(87.7 \mathrm{eV})$ and $\mathrm{C} 1 \mathrm{~s}(285.0 \mathrm{eV})$.

\section{Results of XPS}

Figure 1 shows measurement points on the exited zinc film surface and spatial distribution of XPS spectra of C1s, O1s, Zn2p3/2, Al2p, Zn3p and Si2p. In C1s spectra, two peaks are recognized. The separation energy between the two peaks depends on the location. O1s peak profiles are relatively wide, which were fitted by three peaks. Zn2p3/2 profiles include two peaks. Intensity of peaks depended on the location, and the two peaks had different dependence on the location. The peaks of $\mathrm{B}$ at $\mathrm{Zn} 2 \mathrm{p}_{3 / 2}$, mostly exist at the center region of the substrate. Peaks of Zn3p include Zn3p3/2, Zn3p1/2 and shift peaks of Zn3p3/2 and Zn3p1/2. It is difficult to fit out four peaks of those, clearly. Analysis of element, Zinc, is focused on Zn2p3/2. The peaks of Si2p spectra almost can't exhibit clearly. Al2p spectra showed strong broad single peak. From the viewpoint of correlation, spectra of C1s at peak $A$ and peak $A$ of $Z \mathrm{Zn}_{2} \mathrm{p}_{3 / 2}$ suggest high correlation. And $\mathrm{O} 1 \mathrm{~s}$ and $\mathrm{Al} 2 \mathrm{p}$ also suggest high correlation. However these correlations include spatial different intensity profiles. In next section we show the results of peak analysis.

\section{Analysis of XPS Spectra}

Elements of XPS spectrum from N1 to N9 were exhibited in Figure 1, respectively. Silicon was scarcely, and Si hadn't been fitted. In Figure 2, XPS spectra of C1s, O1s, Zn2p3/2 and Al2p at N5 were fitted out, but Si2p. The 

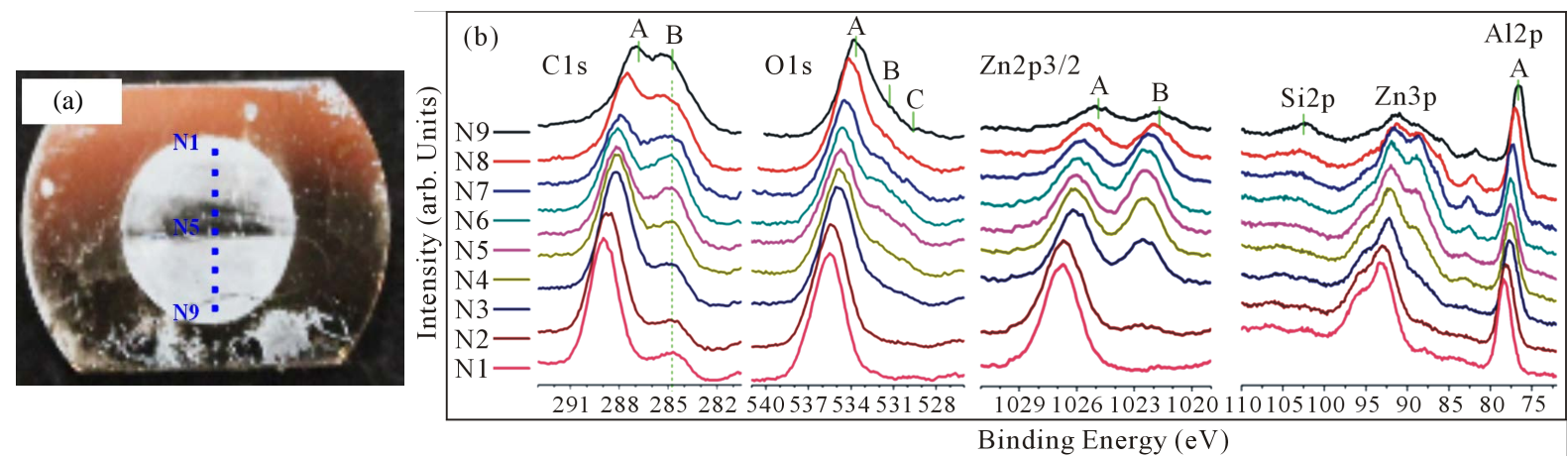

Figure 1. XPS spectrum of C1s, O1s, Zn2p3/2 and Al2p depended after 44 weeks. (a) showed N1-N9 9 points along vertical, and N5 at the center of sample. (b) showed the results of XPS measurements of the 9 points.
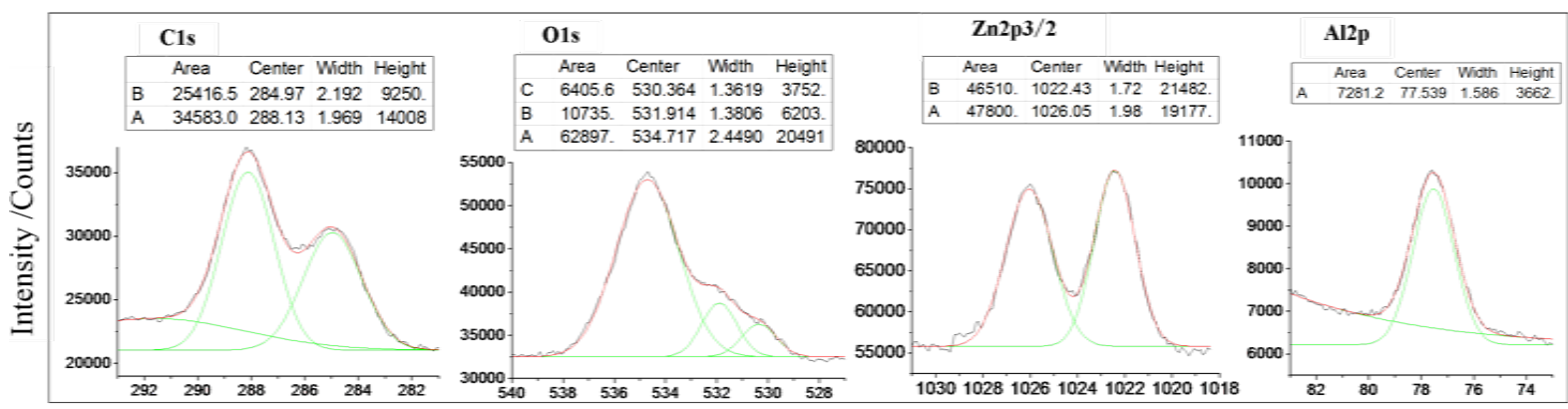

Binding Energy (eV)

Figure 2. XPS spectrum of C1s, O1s, Zn2p3/2 and Al2p at N5 was fitted out.

spitting peaks and characteristic factors were indicated out:

1) C1s was spitted to two (A and B) peaks: Binding energy of peak center of B was $285.0 \mathrm{eV}$, what showed as the factor of "Center" in table of Figure 2-C1s. The standard point of these data is C1s (285.0 eV). The width at half maximum height of peak B was $2.2 \mathrm{eV}$, what exhibited as factor of "Width". The integral value of intensity of peak B was 25416, what exhibited as factor, “Area”. The intensity of peak center of B was 9250, what exhibited as factor, "Height”. The factors of fitted peak A also showed in the table at Figure 2-C1s.

2) O1s was a boarding peak. At high binding energy side, there was a strong satellite marked to A. From $530 \mathrm{eV}$ to $534 \mathrm{eV}$, there should be two different combinative states oxygen, at least. The factors of fitted peaks of oxygen were exhibited in the table of Figure 2-O1s.

3) Spectrum of Zn2p3/2 was fitted to two peaks, clearly. The factors of fitted peaks of zinc were exhibited in the table of Figure 2-Zn2p3/2.

4) Spectrum of Al2p was single. The factors of fitted peaks of alumina were exhibited in the table of Figure 2-Al2p.

The fitting results of binding energy of peaks from N1 to N9 were showed in Figure 3; and the fitting results of intensity of fitting out peaks from N1 to N9 were showed in Figure 4.

Figure 3 showed the spatial change of the binding energy of C1s, O1s, Zn2p3/2 and Al2p from N1 to N9. The peaks at $285.0 \mathrm{eV}$ of C1s are due to adsorbed carbon oxide, which was fixed as the standard of the spectra. C1s at $288 \mathrm{eV}$ region showed stepwise structure. The stepwise structures were observed in all spectra with higher binding energies. The other peaks with lower energies of $\mathrm{O} 1 \mathrm{~s}$ and $\mathrm{Zn} 2 \mathrm{p}_{3 / 2}$ showed monotonous decrease as changing the position from N1 to N9.

Figure 4 showed the spatial profiles of integrated peak intensities of analyzed peaks. Intensity profiles of two peaks at A and B of C1s showed complemented structures with odd symmetry. The summation of these peaks was almost constant. Intensity profiles of O1s include two groups. The symmetry of these profiles is all even symmetry. However, the profiles with lower energy, peak-B and Peak-C of O1s, showed even symmetrical, on the other hand, the profiles with higher energy, peak-A of O1s, showed the sum of even symmetrical and odd symmetrical. Intensity profiles of Zn2p3/2 include even symmetry of the profile with lower energy, peak-B of 

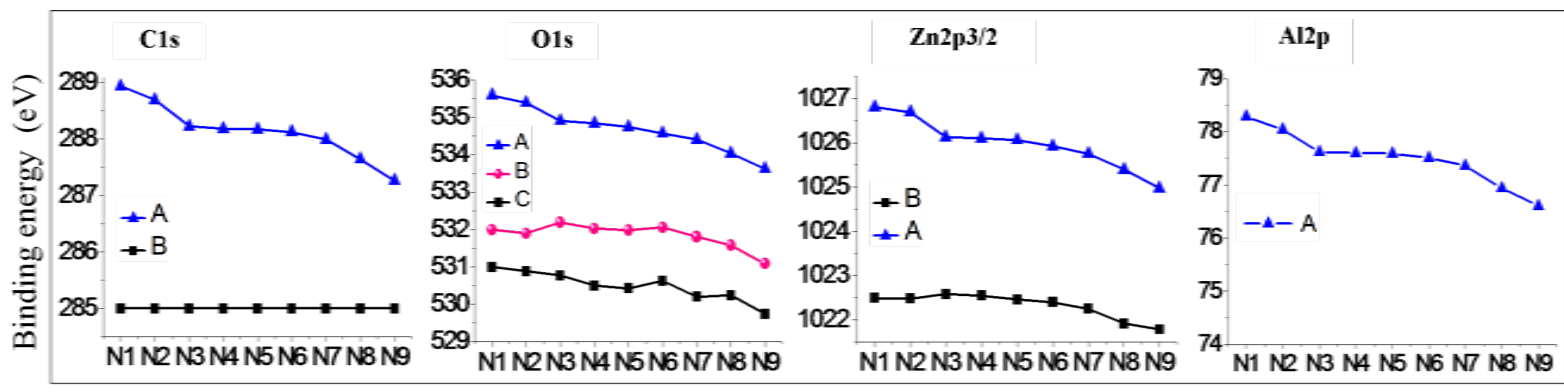

Location

Figure 3. Spatial changes of the binding energy of C1s, O1s, Zn2p3/2 and Al2p.

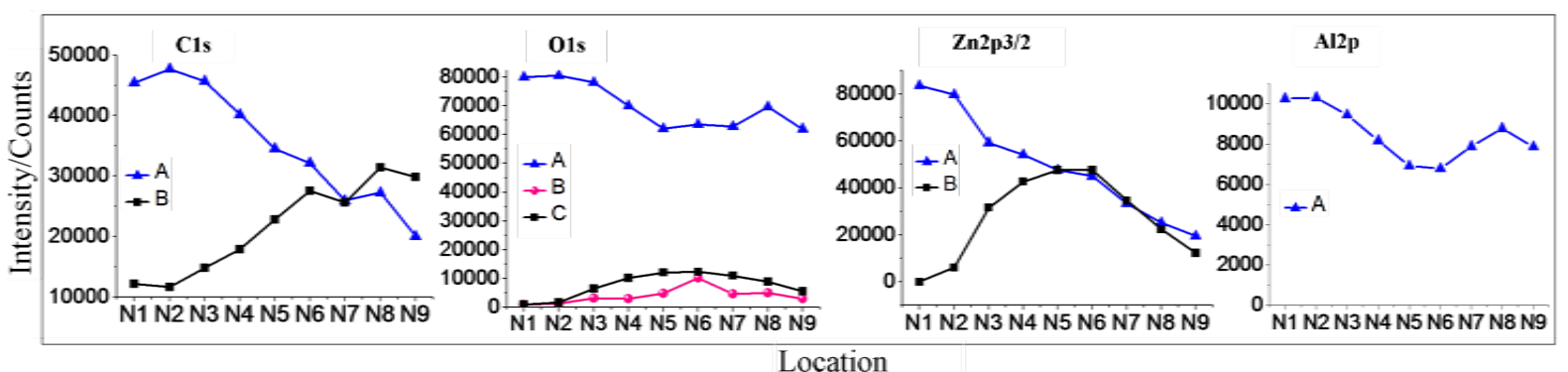

Figure 4. XPS spectrum intensities of C1s, O1s, Zn2p3/2 and Al2p spatial symmetry.

$\mathrm{Zn} 2 \mathrm{p}_{3 / 2}$, and odd symmetry of that with higher energy, peak-A of $\mathrm{Zn} 2 \mathrm{p}_{3 / 2}$. The summation of these is not constant. The intensity in the half from N6 to N9 decreased linearly. The intensity profile of Al2p showed the sum of even symmetrical and odd symmetry at high energy side of normal $\mathrm{Al}_{2} \mathrm{O}_{3}(74.4 \mathrm{eV})$.

\section{Discussion}

We discussed the states of special excited zinc film and the spatial symmetry of zinc, and we calculated the important independence parameters, $\mathrm{U}_{\mathrm{dc}}, \mathrm{T}$, and $\Delta$ of charge transfer mechanism.

\subsection{The States of Zinc (This Part Has Done a Lot of Modifications)}

From Figure 5(b), the XPS spectrum of surface slightly oxidized mental zinc (Zn, ZnO) and N5 in Figure 1 was showed. There were two satellites at the high binding energy side of Zn2p at N5. To elucidated the states of zinc in our special film. Here we consider the 2p XPS of transition mental compounds, firstly. Experimental data observed by Rosencwaig et al. (1971) [6]. There is no satellite peak in the $\mathrm{Zn2p}$ of $\mathrm{ZnF}_{2}$ where the $3 \mathrm{~d}$ shell is filled. However, the satellite of $2 \mathrm{p}$ XPS from $\mathrm{CuF}_{2}$ to $\mathrm{MnF}_{2}$ occurs on the higher binding energy side of the main peak. It is now well established that it originates from the charge transfer between the ligand $2 \mathrm{p}$ and metal $3 \mathrm{~d}$ orbital. From other researches, the properties of strong correlation and high temperature superconductivity directly related to half filled $3 \mathrm{~d}$ orbital of the transition metals. However, how can distinguish the $3 \mathrm{~d}$ full filled states and 3d half filled state? Figure 5(a) showed X-ray photo spectroscopy of Cu2p and $\mathrm{O} 1 \mathrm{~s}$ in $\mathrm{Cu}, \mathrm{Cu} 2 \mathrm{O}$ and $\mathrm{CuO}$ [7]-[9]. Metal $\mathrm{Cu}$ and $\mathrm{Cu}_{2} \mathrm{O}$ with $3 \mathrm{~d}^{10}$ showed almost the same spectrum of $\mathrm{Cu} 2 \mathrm{p}$, but $\mathrm{CuO}$ with $3 \mathrm{~d}^{9}$ (orbital of $3 \mathrm{~d}$ is not full filled.) showed different spectrum of $\mathrm{Cu} 2 \mathrm{p}$ to $\mathrm{Cu}$ and $\mathrm{Cu}_{2} \mathrm{O}$. The peak at high binding energy side was identified as the peak, what due to $2 \mathrm{p}^{5} 3 \mathrm{~d}^{9}$ final state that the movement of the holes did not occur. The peak of low binding energy side could be fitted to two peaks. The fitting peak at high binding energy side is final state of $2 \mathrm{p}^{5} 3 \mathrm{~d}^{10} \mathrm{~L}$, what the movement of hole was occur from Cu3d to O2p with the photoelectron emission. Components what form the sharp rise at the low binding energy side, is $2 \mathrm{p}^{5} 3 \mathrm{~d}^{10}$ final state, that is derived from a hole in the valence band is moved to O2p band of adjacent site.

However, $3 \mathrm{~d}$ orbit of $\mathrm{ZnO}$ and $\mathrm{Zn}$ is full filled; and new peak at high energy side isn't exhibited. The electron transition from O2p to Zn3d couldn't happen, and there is no satellite peaks to identify the $3 \mathrm{~d}^{10} \underline{\mathrm{L}}$ state of high binding energy side in the $\mathrm{X}$-ray photoelectron spectroscopy. If $3 \mathrm{~d}$ is half filled, new peaks at the high binding 


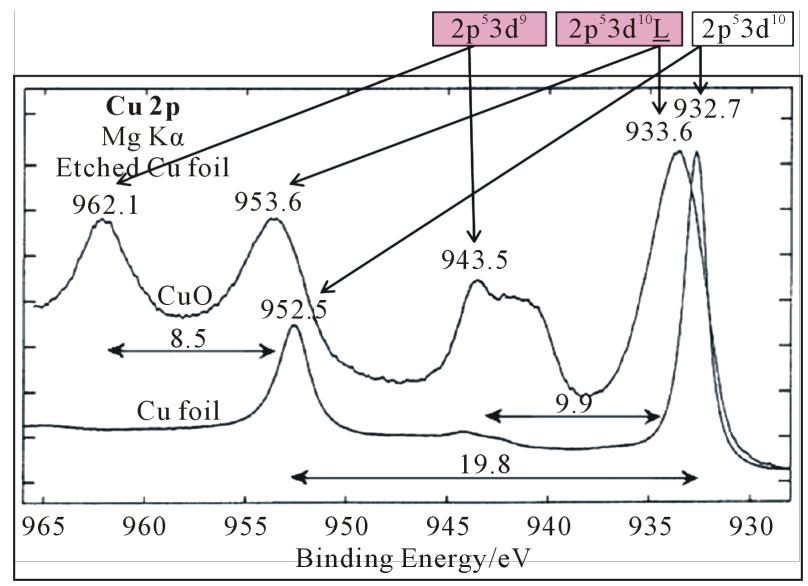

(a)

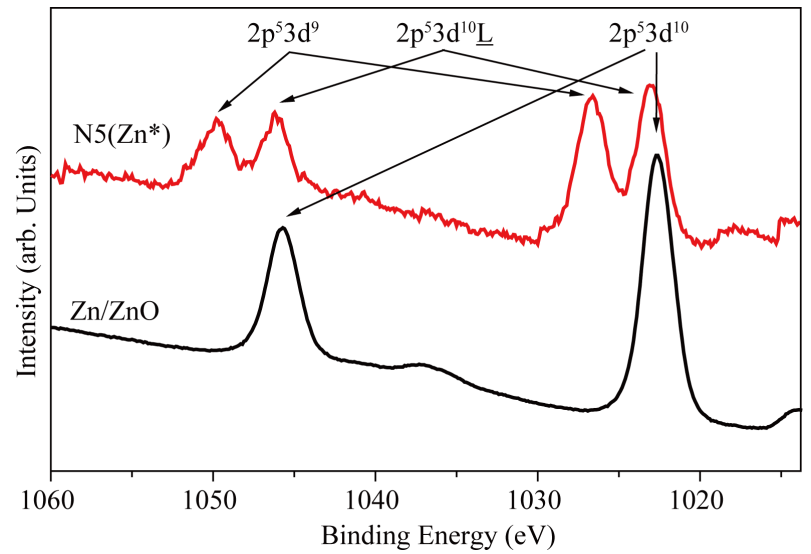

(b)

Figure 5. (a) 2p XPS spectrum of $\mathrm{Cu}$ foil and $\mathrm{CuO}$ [8] [9]; (b) 2p XPS spectrum of $\mathrm{Zn} / \mathrm{ZnO}$ and $\mathrm{N}_{5}\left(\mathrm{Zn}^{*}\right), \mathrm{N}_{5}$ in Figure 1 at center of the sample ( $\mathrm{Zn}^{*}$ : Excited state of zinc).

energy side could be exhibited, what is caused by electron transition between ligand to transition metal. From this we can infer that, in the Figure 1, Figure 2 and Figure 5(b), the new peaks at the high binding energy side of $\mathrm{Zn}^{*}$ could be exhibited, what caused by half filled of $\mathrm{Zn}^{*} 3 \mathrm{~d}^{\mathrm{n}}(\mathrm{n}<10)$ orbits. We considered the peak A of

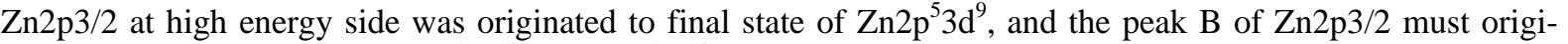
nated to the final states of $\mathrm{Zn} 2 \mathrm{p}^{5} 3 \mathrm{~d}^{10}$ or $\mathrm{Zn} 2 \mathrm{p}^{5} 3 \mathrm{~d}^{10} \mathrm{~L}$. In other experiments, the relative intensity of peak $\mathrm{A}$ and peak $B$ was changed by irradiation of strong $X$-ray, and it was reported between $3 \mathrm{~d}^{9}$ and $3 \mathrm{~d}^{10} \underline{\mathrm{L}}$ [6] [7] [10]-[13].

We considered peak A of $\mathrm{Zn} 2 \mathrm{p}_{3 / 2}$ originated by final state of $\mathrm{Zn} 2 \mathrm{p}^{5} 3 \mathrm{~d}^{9}$, peak B of $\mathrm{Zn} 2 \mathrm{p}_{3 / 2}$ originated by the final state of $\mathrm{Zn} 2 \mathrm{p}^{5} 3 \mathrm{~d}^{10} \underline{\mathrm{L}}$.

\subsection{The Spatial Symmetry of Excited Zinc Film}

The correlation between analyzed profiles is useful to decide the interaction between each element. From Figure 4, the electron state of peak-A in C1s correlates with the electron state of peak-A in Zn2p3/2. The electron state of Al2p correlates with the electron state of peak-A of O1s. The state of peak-C in O1s correlates with peak-B of Zn2p3/2. These correlations suggest the combination of the origins of these states. The satellites at high binding energy side of zinc were odd symmetry, and the peaks at low binding energy side of zinc were even symmetry. We can realize that the initial state of $\mathrm{Zn3d}$ is odd symmetry, but the initial state of $\mathrm{Zn}^{9} \mathrm{~d}^{10} \underline{\mathrm{L}}$ shows even sym-

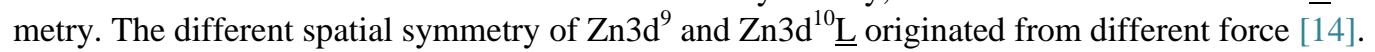

The external fields on earth just gravitational field, magnetic field and electric field can be considered. From sample preparation, there is an even symmetry electric field be made out by electron incident. The even symme- 
try of $\mathrm{Zn} 3 \mathrm{~d}^{10} \underline{\mathrm{L}}$ correlated to electric field. There was micro electric current occurred on the sapphire substrate, because of electron incident, and the direction of the micro electric currents were same. The same direction micro electric current made the same direction of magnetic field. So, the odd symmetry of Zn3d9 could correlate to magnetic field.

\subsection{Theoretical Calculation of CT (This Part Has Done a Lot of Modifications)}

The correspondence between initial state, final state and XPS spectrum of charge transfer process was showed in Figure 6. Factors of charge transfer energy $(\Delta)$, mixing energy $(\mathrm{T})$ and coulomb interaction energy between $3 \mathrm{~d}$ and inner holes $(\mathrm{Q})$ are the most important factors in charge transfer process. In Figure 6, we show an ionic state energy diagram of XPS. From charge transfer theory by A. Kotani and K. Okada [7] [15] [16], there are initial state of $d^{n}$ and $d^{n+1} \underline{L}(n<10)$, and energy of $d^{n+1} \underline{L}$ state is $\Delta e V$ bigger than $d^{n}$ state. Mixed valence state of $\mathrm{d}^{\mathrm{n}}$ is marked as "G.S.", and energy is smaller than $\mathrm{d}^{\mathrm{n}}$ of initial state. The final state originated to emission of inner core electroscopes. If energy of $\underline{\underline{c}} \mathrm{~d}^{\mathrm{n}}=\mathrm{E}_{0}$, energy of $\underline{\underline{c}} \mathrm{~d}^{\mathrm{n}+1} \underline{\underline{L}}$ equal $\mathrm{E}_{0}+\Delta-\mathrm{Q}$.

About $\mathrm{T}, \Delta, \mathrm{U}_{\mathrm{dd}}$ and $\mathrm{Q}$, the following series of equations is established:

1) Ground state and final state showed as following:

$$
\begin{gathered}
\text { G.S. }|\mathrm{g}\rangle=\cos \theta_{\mathrm{g}}\left|\mathrm{d}^{9}\right\rangle-\sin \theta_{\mathrm{g}}\left|\mathrm{d}^{10} \mathrm{t}\right\rangle \tan \theta_{\mathrm{g}}=\frac{\mathrm{E}_{\mathrm{g}}}{\mathrm{T}}=\frac{\sqrt{\Delta^{2}+4 \mathrm{~T}^{2}}-\Delta}{2 \mathrm{~T}} \\
\text { F.S. }\left\{\begin{array} { l } 
{ | f _ { 1 } \rangle = \operatorname { c o s } \theta _ { f } | \mathrm { d } ^ { 9 } \rangle - \operatorname { s i n } \theta _ { f } | \mathrm { d } ^ { 1 0 } \mathrm { t } \rangle } \\
{ | f _ { 2 } \rangle = \operatorname { c o s } \theta _ { f } | \mathrm { d } ^ { 9 } \rangle + \operatorname { c o s } \theta _ { f } | \mathrm { d } ^ { 1 0 } \mathrm { E } \rangle }
\end{array} \left\{\begin{array}{l}
\tan \theta_{f}=\frac{\sqrt{\Delta_{f}^{2}+4 \mathrm{~T}^{2}}-\Delta_{f}}{2 \mathrm{~T}} \\
\Delta_{f}=\Delta-\mathrm{Q}
\end{array}\right.\right.
\end{gathered}
$$

2) The peak state energy of $\mathrm{f}_{1}$ and $\mathrm{f}_{2}$, peak interval of $\delta \mathrm{E}_{\mathrm{B}}$, the intensity ratio of peaks showed as:

$$
\begin{aligned}
& \text { Final state energy }: \mathrm{E}_{f 1,2}=\frac{\Delta_{f} \pm \sqrt{\Delta_{f}^{2}+4 \mathrm{~T}^{2}}}{2} \\
& \text { Peak interval }: \delta \mathrm{E}_{\mathrm{B}} \equiv \mathrm{E}_{f 2}-\mathrm{E}_{f 1}=\sqrt{\Delta_{f}^{2}+4 \mathrm{~T}^{2}} \\
& \text { Intensity ratio }: \mathrm{R}=\frac{\mathrm{I}_{f 2}}{\mathrm{I}_{f 1}}=\tan ^{2}\left(\theta_{f}-\theta_{\mathrm{g}}\right)
\end{aligned}
$$

From Figures 1-3, we got the peak intervals and intensity ratio from source data of XPS. In our zinc film, the ligand should be $\mathrm{C}$. The value of $\mathrm{Q}$ is not very dependent on the metal ions, and $\mathrm{Q}$ is reduced with electrical negative degrees of ligand to transition metal compound [7] [15]. In this calculation, we supposed $\mathrm{Q}=5.5 \mathrm{eV}$, equal coulomb interaction energy of $d-d, U_{d d}$.

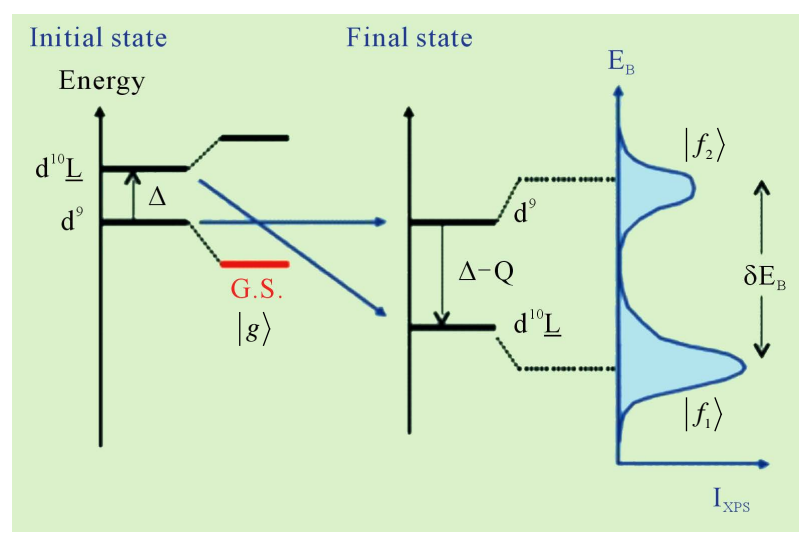

Figure 6. The correspondence between initial state, final state and XPS spectrum of charge transfer process [15] L: An electron transited from ligand to metal, where must be hole exist. 
Table 1. The independent parameters of $\mathrm{U}_{\mathrm{dc}}, \Delta$ and $\mathrm{T}$ were calculated from N1 to N9.

\begin{tabular}{|c|c|c|c|c|c|c|c|c|c|c|c|c|c|c|c|}
\hline \multicolumn{11}{|c|}{ The independent parameters $\left(\Delta, \mathrm{T}, \mathrm{U}_{\mathrm{dc}}\right)$ of $\mathrm{Zn}^{*}$ films } & \multicolumn{3}{|c|}{ Ground state } & \multicolumn{2}{|c|}{ Final state } \\
\hline & $\delta \mathrm{E}_{\mathrm{B}}$ & $\mathrm{I}_{+} / \mathrm{I}_{-}$ & $\mathbf{U}_{\mathrm{dc}}$ & Udd & $\Delta$ & $\Delta \mathrm{f}$ & $\left(\theta_{\mathrm{f}}\right)$ & $(\theta g)$ & $\mathbf{T}$ & Eg & $\mathrm{d}^{9}$ & $\mathrm{~d}^{10} \underline{\mathrm{L}}$ & $2 p^{5} d^{9}$ & $2 \mathrm{p}^{5} \mathrm{~d}^{10} \underline{\mathrm{L}}$ & $2 p^{5} d^{10} \underline{L}+E g$ \\
\hline $\mathrm{N} 1$ & 4.31 & 60 & 5.5 & 5.5 & 1.20 & -4.30 & 87.35 & 4.72 & 0.10 & -0.01 & 0.00 & 1.20 & 0.00 & -4.30 & -4.31 \\
\hline N2 & 4.20 & 7.31 & 5.5 & 5.5 & 1.34 & -4.16 & 81.72 & 12.28 & 0.31 & -0.07 & 0.00 & 1.34 & 0.00 & -4.16 & -4.23 \\
\hline N3 & 3.55 & 1.78 & 5.5 & 5.5 & 2.23 & -3.27 & 68.82 & 15.82 & 0.69 & -0.19 & 0.00 & 2.23 & 0.00 & -3.27 & -3.46 \\
\hline N4 & 3.55 & 1.19 & 5.5 & 5.5 & 2.36 & -3.14 & 64.98 & 17.54 & 0.83 & -0.26 & 0.00 & 2.36 & 0.00 & -3.14 & -3.40 \\
\hline N5 & 3.60 & 0.91 & 5.5 & 5.5 & 2.43 & -3.08 & 62.48 & 18.87 & 0.94 & & 0.00 & 2.43 & 0.00 & -3.08 & -3.40 \\
\hline N6 & 3.52 & 0.92 & 5.5 & 5.5 & 2.50 & -3.00 & 62.39 & 18.18 & 0.92 & -0.30 & 0.00 & 2.50 & 0.00 & -3.00 & -3.30 \\
\hline N7 & 3.50 & 0.91 & 5.5 & 5.5 & 2.54 & -2.96 & 61.85 & 18.22 & 0.94 & -0.31 & 0.00 & 2.54 & 0.00 & -2.96 & -3.27 \\
\hline N8 & 3.47 & 1.05 & 5.5 & 5.5 & 2.50 & -3.00 & 63.22 & 17.52 & 0.88 & -0.28 & 0.00 & 2.50 & 0.00 & -3.00 & -3.28 \\
\hline N9 & 3.19 & 1.55 & 5.5 & 5.5 & 2.67 & -2.83 & 65.28 & 14.40 & 0.73 & -0.19 & 0.00 & 2.67 & 0.00 & -2.83 & -3.02 \\
\hline
\end{tabular}

From the energy difference, $\delta \mathrm{E}_{\mathrm{B}}$, and peak intensity ratio, $\mathrm{I}_{+} / \mathrm{I}_{-}$, between $2 \mathrm{p}^{5} 3 \mathrm{~d}^{9}$ and $2 \mathrm{p}^{5} 3 \mathrm{~d}^{10} \mathrm{~L}$, the $\Delta$ and $\mathrm{T}$ were estimated. The results were exhibited in Table 1 . In the region where the intensity of $2 \mathrm{p}^{5} 3 \mathrm{~d}^{10} \mathrm{~L}$ becomes large, $\Delta$ becomes small, $1.2 \mathrm{eV}<\Delta<2.7 \mathrm{eV}$, and T becomes small, too, $0.1 \mathrm{eV}<\mathrm{T}<0.9 \mathrm{eV}$, respectively.

\subsection{The Structural Instability of Excited Zn Film}

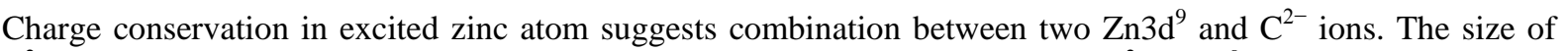
$\mathrm{C}^{2-}$ is almost comparable to that of oxygen atom. If the electrons transfer from $\mathrm{C}^{2-}$ to $3 \mathrm{~d}^{9}$, the size of the carbon shrinks to $0.154 \mathrm{~nm}$ from $0.25 \mathrm{~nm}$. Therefore the charge transfer induces a vacancy due to the rapid diffusion of carbon. In our experiment, the sample showed high mobility of the constituting elements.

\section{Conclusions}

This paper presents a result of the challenge of the excitation of inner-core electron system with long lifetime of excited zinc films. The advanced zinc films with excited inner-core electron, $3 \mathrm{~d}^{9}$, were formed by surface ion recombination process controlled by the collision energy. We report experimental results of XPS measurements of 9 points along vertical direction from N1 to N9. The most pronounced futures are existence of the satellites,

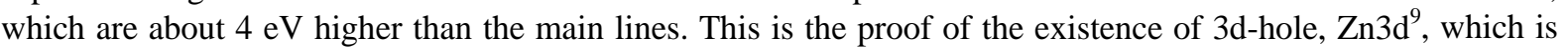
the same mechanism of $\mathrm{CuO}$. The measured XPS spectra apparently depend on the location in the sample. These

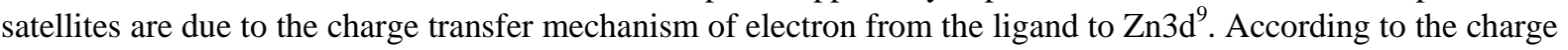
transfer mechanism proposed by A. Kotani and K. Okada, it was clarified that the origins of these peaks are $\underline{c} 3 \mathrm{~d}^{10} \underline{\mathrm{L}}$ for the main peak and $\underline{\mathrm{c}} 3 \mathrm{~d}^{9}$ for the satellite, respectively. The samples contain $\mathrm{Zn}, \mathrm{Al}, \mathrm{O}, \mathrm{C}$ and $\mathrm{Si}$. The surface densities of these elements depend on the location of the sample. In the analysis along the vertical direc-

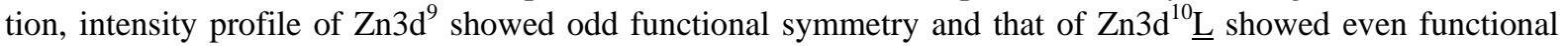
symmetry. Only the intensity profile of C1s $(288 \mathrm{eV})$ showed the same spatial correlation with Zn3d $\mathrm{d}^{9}$. This is the reason that the ligand for ${\mathrm{Zn} 3 \mathrm{~d}^{9}}$ is Carbon, not Oxygen. The other elements roughly show the even functional symmetry. We analyzed experimental data by using Kotani and Okada's theoretical model. From the energy difference, $\delta \mathrm{E}_{\mathrm{B}}$, and peak intensity ratio, $\mathrm{I}_{+} / \mathrm{I}_{-}$, between $2 \mathrm{p}^{5} 3 \mathrm{~d}^{9}$ and $2 \mathrm{p}^{5} 3 \mathrm{~d}^{10} \mathrm{~L}$, the energy for charge transfer, $\Delta$, and mixing energy, T, were estimated. In the region where the intensity of $2 \mathrm{p}^{5} 3 \mathrm{~d}^{10} \underline{\mathrm{L}}$ becomes large, $\Delta$ becomes small, $1.2<\Delta<2.7$, and $\mathrm{T}$ becomes small, too, $0.1<\mathrm{T}<0.9$, respectively. In this calculation, we supposed $\mathrm{U}_{\mathrm{dc}}$ $=5.5 \mathrm{eV}$. The excited zinc films also showed high mobility of the constituting elements which correlated to $\mathrm{C}^{2-}$.

We conclude that the excited zinc films have $3 \mathrm{~d}$-hole, $3 \mathrm{~d}^{9}$, with almost permanent lifetime and $3 \mathrm{~d}^{9}$ combine with carbon. Although the detailed mechanism of the long lifetime of the inner-core excited zinc atoms is not clear at the present time, this material suggests the high potential for the advanced application in wide area [17].

\section{References}

[1] Hay, P.J., Dunning Jr., T.H. and Raffenetti, R.C. (1976) Journal of Chemical Physics, 65, 2679. 
[2] Wei, M., Boutwell, R.C., Garrett, G.A., Goodman, K., Rotella, P. and Schoenfeld, W.V. (2013) Material Letters, 97, 11-14. http://dx.doi.org/10.1016/j.matlet.2013.01.090

[3] Hamasaki, M., Yamaguchi, M., Kuwayama, M. and Obara, K. (2011) A New Approach for Sustainable Energy Systems Due to the Excitation of Inner-Core Electrons on Zinc Atoms Induced by Surface-Ion-Recombination. AIP Conference Proceeding, 1415, 43-50. http://dx.doi.org/10.1063/1.3667216

[4] Obara, M., Hamasaki, M., Manaka, H. and Obara, K. (2011) Slowly Relaxing Structural Defects of Zinc Films with Excited States Induced by Ion Recombination Processes. Advanced Materials Research, 277, 11-20. http://dx.doi.org/10.4028/www.scientific.net/AMR.277.11

[5] Callis, P.R. and Scott, T.W. (1983) Perturbation Selection Rules for Multiphoton Electronic Spectroscopy of Neutral Alternant Hydrocarbonsa). Journal of Chemical Physics, 78, 16. http://dx.doi.org/10.1063/1.444537

[6] Rosencwaig, A., Wertheim, G.K. and Guggenheim, H.J. (1971) Origins of Satellites on Inner-Shell Photoelectron Spectra. Physical Review Letters, 27, 479. http://dx.doi.org/10.1103/PhysRevLett.27.479

[7] Rosencwaig, A. and Wertheim, G.K. (1971) Origins of Satellites on Inner-Shell Photoelectron Spectra. Physical Review Letters, 27, 479-481. http://dx.doi.org/10.1103/PhysRevLett.27.479

[8] Tahir, D. and Tougaard, S. (2012) Electronic and Optical Properties of $\mathrm{Cu}, \mathrm{CuO}$ and $\mathrm{Cu}_{2} \mathrm{O}$ Studied by Electron Spectroscopy. Journal of Physics: Condensed Matter, 24, 175002. http://dx.doi.org/10.1088/0953-8984/24/17/175002

[9] Tanaka, K. (1998) X-Ray Photoelectron Spectroscopy.

[10] Schon, G. (1973) ESCA studies of $\mathrm{Cu}, \mathrm{Cu}_{2} \mathrm{O}$ and CuO. Surface Science, 35, 96-108. http://dx.doi.org/10.1016/0039-6028(73)90206-9

[11] Tsuda, N., Nasu, K. and Fujimori, A. (2010) Electronic Conduction in Oxides. Heidelberg. 223.

[12] Schon, G. (1973) Auger and Direct Electron Spectra in X-Ray Photoelectron Studies of Zinc, Zinc Oxide, Gallium and Gallium Oxide. Journal of Electron Spectroscopy and Related Phenomena, 2, 75-86. http://dx.doi.org/10.1016/0368-2048(73)80049-0

[13] de Groot, F. and Kotani, A. (2008) Core Level Spectroscopy of Solids, 1-40, 71-75, 145-160, 182-185.

[14] Fazelzadeh, S.A. and Ghavanloo, E. (2012) Nonlocal Anisotropic Elastic Shell Model for Vibrations of Single-Walled Carbon Nanotubes with Arbitrary Chirality. Composite Structures, 94, 1016-1022. http://dx.doi.org/10.1016/j.compstruct.2011.10.014

[15] Okada, K. (2004) Changes in the Electronic State of the Copper Oxide and Cu 2p XPS by Doping.

[16] Veal and Paulikas (1985) Final-State Screening and Chemical Shifts in Photoelectron Spectroscopy. Physical Review B, 31, 5399. http://dx.doi.org/10.1103/PhysRevB.31.5399

[17] Yan, S.C., Yu, H., Wang, N.Y., Li, Z.S. and Zou, Z.G. (2011) ESI for Chemical Community. 